THEORIA ET HISTORIA S CI EN TIA RUM, V O L . VII, N²

Ed. Nicolas Copernicus University 2003

\title{
Douwe Draaisma (2000) Metaphors of Memory: A History of Ideas about the Mind. Cambridge University Press. ISBN 0521650240
}

\section{Metaphors of the human mind, if not of human memory}

A finely illustrated 'history of ideas about the mind' this certainly is, though more a book concerned with the metaphors, than with the essence, of human memory per se. What it does superbly well, but not explicitly, is to tell us why this topic is important to the philosophy of mind and consciousness.

Previously available only in the Dutch language (as 'De Metaforenmachine - een geschiedenis van het geheugen', by Historische Uitgeverij, 1995) Draaisma's English version as reviewed here indeed provides us with an historical tale of 'metaphors of memory' running through time from Plato's tablets to the electronic neural networks of recent times. The material covered is itself a brief natural history museum case of ideas concerned principally with our thoughts on human memory (and memory processes) in away reminiscent of Gregory's (1981) 'Mind In Science', especially with reference to the use of explanations derived from our understanding of the workings of man-made technological developments. This new book, blends Gregory's thesis together with that of the desires of Pribram's (1971) Languages of the Brain, timely bringing us into the $21^{\text {st }}$ Century for a view and review of the recurrent metaphorical landscapes with regards our understanding of the phenomenon of human memory.

Much of Douwe Draaisma's own thesis is, however, concerned with our w/junderstanding the phenomenon of human memory; his critical points (rightly in my view) often aimed at the repeated abstraction mistakes made by those coming to champion the various explanations of memory as have been offered throughout recorded history. Moving sequentially in time, we are introduced to metaphors familiar and unfamiliar, from wax-impressions, through pigeon-holes and libraries of books, and via photography and holograms to computer hard-disks and neural nets. One very valuable insight provided by Draaisma's thesis is that, despite the often quite radical changes in technology giving 
rise to each of the subsequent metaphorical replacements used to explain memory processes, common to most of them is the notion that although such devices do indeed serve as memory systems of a sort, they do not exist as such without brains/observers to decode them. As such, all of the metaphorical claims as have been made at various times have continued to fail as models of instantiated human physiological memory system(s) - either as process or principle. Furthermore, few, if any, were to lead to any new neurophysiological findings or questions as a result, remaining instead firmly attached to the logic of memory processes, not to its ellucidation with regards physiological mechanisms. In reading this volume, one is repeatedly struck by the finding that neurophysiology was largely being ignored following the introduction of each notion comparing the 'mechanics of the mind' (in this case memory) to the mechanisms of everyday objects in order to understand (by mensuration) how they might operate. With respect to the more philosophical attitudes taken with each development, it is also noteworthy that both technical innovation and theoretical (conceptual) advances were rarely made by the same individual.

However, Draaisma's book is well balanced with respect to his inclusion of these topics throughout the nine chapters, telling his story fluently and with consistent rhythm. If I were to fault the choice of historical landmarks/events to be reported, I would comment upon the relative allocation of time and space devoted to definable eras. For example, having dispensed with the Graeco-Roman contributions in the first (relatively short) chapter, we jump almost a thousand years to the time of the printing and the middle- ages' monasticism. One cannot help wondering whether there might be another, yet untold story, belonging to the near, middle or far eastern scholastic traditions which might brought to bear upon this story? If the advent of the printing press was such a significant factor in 'the enlightenment' and the European return towards inward psychological reflection, one is left pondering why, for example, the Chinese culture (possessive of the printing press some 400 years earlier) did not make a similar advance ? Perhaps such comparative study is beyond the remit of this volume, but whether it would perhaps read differently if not exclusively a history of Western ideas concerned with memory, I would be interested to know.

The middle chapters discuss the light being shed upon the mystery of memory following the discoveries of phosphorescence and the first experiments with photography and later, cinematography. In this era, the artifacts produced were at last seemingly more congruent with (the intuitively human) recall of both stationary and dynamic images as visible to the naked eye. But although any clear neurophysiology of vision was still a long way off for the future, we are reminded that at this time many thinkers (forgetting who might be able to view them !) were beginning to argue that such images could somehow be frozen "into a motionless neuronal trace".

The final four chapters are set firmly in the $20^{\text {th }}$ Century, taking us from the earliest electrical devices, via the telephone exchange, to the modern electronic, binary computer metaphors of mind. In this latter half of the book, we come to realise that whereas the Behaviourists (and later even Turing himself) regarded their 'intelligent machines' as 
essentially anti-mentalist, others such as Hull proposed that an exact copy of the human nervous system would produce a 'truly psychic machine'. This taking of the computer metaphor from behaviourism (the dominant focus for the first 50 or so years of American psychology) was to give birth to a new field of psychology, that of cognitive science. It was here that one saw an interesting interchange of vocabulary develop between the studies of human memory and that of computer science. One talked for the first time, for example, using such terms as input, readin, encoding, 'back-up memory', storage, address, retrieval, output etc.., in common parlance. However, even with this, such an enriched differentiable new language, the rush to produce 'black box ' models of human memory processes remained abstract and incomplete - and notably without clear existence proofs in reality, or the demonstration of supporting physiological substrates. This point is alluded to in several places throughout the book, but could have been made stronger as the occasion allowed. However, a further issue that gains no mention, is the similar case of the software designer (including some of the very good ones) who may nonetheless seem to be capable of quite remarkable feats of programming/modeling, yet have no idea concerning the physical mechanisms of the processor chips they are operating ! The reason for mentioning this point here is to illustrate the point that even our most convincing neural network programs, at very best, remain merely capable of reproducing the phenomena they are designed to explain, rather than providing the explanations required of them. As such, even our most up-to-date neural net connectionist model - nay, 'metaphors of brain' - remains a redescription of the problem, its heuristic value firmly fixed at the conceptual level of description. Whether the reactivation of the human brain's neuronal tract(s) following a particular object/event representation is actually a memory (in the sense of being an experience of an event having occurred in the past) or simply a repetition of the initial experience to be remembered (vis-a-vis neural networks) remains open for future empirical experiment and analysis to determine. Maybe our next 'metaphor of memory' will be better able to inform us on this issue?

A. R. Dickenson*

\section{References}

Gregory, R. L. (1981) Mind In Science London: Wiedenfeld \& Nicholson.

Pribram, K. H. (1971) Languages of the Brain. Englewood Cliffs, NJ: Prentice-Kail.

* Address for correspondence: Dept. of Anatomy \& Neurobiology, Washington University School of Medicine, Box 8108, 660 S. Euclid Ave., St. Louis, MO 63110, USA. (314) 7474095. http:// eye-hand.wustl.edu/lab/people/dickinson.html 